\title{
Influencia de la aparatología ortodóntica sobre la ocurrencia de Candida spp. en la cavidad oral
}

\author{
Sandra E. Hernández-Solís, Florencio Rueda-Gordillo, Alan D. Flota-Alcocer, Fernando J. Aguilar-Ayala, \\ María del S.C. Rodríguez-Fernández y Esperanza M. Lama-González
}

\section{Influence of orthodontic appliances on the occurrence of Candida spp. in the oral cavity}

Introduction: Use of orthodontic appliances creates favorable conditions for growth of dental bacterial plaque. Aim: To establish if use of orthodontic appliance affects the presence of Candida species in the oral cavity in a group of pediatric patients. Materials and Methods: We studied 60 patients between 4 and 10 years old, requiring treatment with orthodontic appliances. Samples were taken of the oral cavity before orthodontic treatment and six months after use began. Samples were inoculated in Sabouraud Dextrose Agar and Candida CHROMagar ${ }^{\circledR}$. Candida species confirmation and identification was done by multiple PCR, and results analyzed with a chi-squared test. Results: Candida presence was greater $(p<0,001)$ in the samples taken six months after use began than in the initial samples. Before treatment, the most frequently found species (percentage of studied patients) was $C$. albicans $(8.3 \%)$, while after treatment it was C. tropicalis $(20.0 \%)$. Conclusions: Use of orthodontic appliances in children increases Candida species presence in the oral cavity. Preventative measures should be taken to avoid oral candidiasis, particularly in immunosuppressed patients.

Key words: Children; oral Candida; orthodontic appliances; carriers.

Palabras clave: Niños, Candida oral, aparatología de ortodoncia, portadores.

\section{Introducción}

$\mathrm{E}$ n la cavidad oral existen condiciones para que los hongos oportunistas del género Candida puedan colonizar la mucosa; sin embargo, la microbiota residente y la respuesta inmune, entre otros, mantienen un equilibro microbiano que evita que los microorganismos expresen factores de virulencia que modulan o favorecen la patogenicidad y se conviertan en patógenos ${ }^{1,2}$.

Se han descrito diversos factores predisponentes para la colonización por Candida, entre los que se encuentran la hospitalización, cáncer, diabetes mellitus, infección por VIH en etapa SIDA y edad avanzada, entre otros ${ }^{1,3-5}$. Asimismo, se ha descrito que la presencia de aparatos bucales de ortodoncia tiene como consecuencia la acumulación de biopelícula dental y modificación del entorno ecológico de la cavidad oral, por lo que puede contribuir a un aumento en la frecuencia de colonización por Candida albicans ${ }^{6-10}$.

En ortodoncia, la aparatología fija y removible, son herramientas esenciales en el tratamiento de los pacientes. Sin embargo, su uso influye en el incremento de la placa dento-bacteriana, debido a la dificultad que implica el llevar a cabo un adecuado aseo oral ${ }^{11,12}$.

La adhesión y colonización de C. albicans es el paso inicial para la candidiasis oral ${ }^{7}$. Aunque otras especies del género Candida han sido asociados como agentes etiológicos de candidiasis oral, C. albicans es la especie más frecuente ${ }^{3}$. Al alterar el entorno ecológico oral, la presencia de aparatología ortodóntica puede inclinar la balanza a favor de la presencia de especies de levaduras ${ }^{7,13}$.

Las maloclusiones, son consideradas el tercer problema de salud oral más frecuente, lo que ha generado en los últimos años un incremento en los tratamientos de ortodoncia para la corrección de estas anomalías ${ }^{8,14}$. Tradicionalmente, al paciente con ortodoncia se le ha considerado como un paciente de bajo riesgo, y al mismo tiempo, los procedimientos ortodónticos como no invasores ${ }^{6,8,14}$.

Las especies de Candida son frecuentemente encontradas en la cavidad oral, con un porcentaje de colonización de hasta $60 \%$ en adultos jóvenes ${ }^{8}$. Estos porcentajes varían de individuo a individuo y según la localización geográfica $^{15}$. Candida albicans es la especie prevalente; no obstante, otras especies, como C. tropicalis, C. glabrata y $C$. parapsilosis, han aumentado su frecuencia en el último tiempo ${ }^{3}$.

Hasta la fecha, son pocos los estudios que han investigado el efecto de la aparatología ortodóntica sobre la portación de Candida en la cavidad oral. Asimismo, todavía existe controversia sobre si los aditamentos ortodónticos utilizados influyen en la colonización oral de Candida, al ser utilizados durante un período de tiempo prolongado ${ }^{9}$. En base a lo anterior, el objetivo del presente trabajo fue
Universidad Autónoma de Yucatán, Mérida, Yucatán, México.

Facultad de Odontología, Departamento de Microbiología Oral y Biología Molecular (SEH, FR, $A D F, M S C R, E M L$ ).

Maestría en Odontología Infantil (FJA, ADF).

Financiamiento: Programa de Apoyo e Impulso a la Investigación de la Facultad de Odontología (PAllFO)

Los autores declaran la ausencia de conflictos de interés.

Recibido: 18 de septiembre de 2015

Aceptado: 5 de mayo de 2016

Correspondencia a: Florencio Rueda-Gordillo gordillo@correo.uady.mx 
evaluar si el uso de aparatología ortodóntica influye en la presencia en cavidad oral de especies de Candida en un grupo de pacientes infantiles.

\section{Material y Métodos}

\section{Pacientes}

Se estudiaron 60 pacientes entre 4 y 10 años de edad, que requirieron tratamiento con aparatología ortodóntica en la Clínica de la Maestría en Odontología Infantil de la Facultad de Odontología de la Universidad Autónoma de Yucatán.

\section{Criterios de inclusión}

Ausencia de enfermedades sistémicas, sin tratamiento previo de ortodoncia, no haber recibido medicación con antimicrobianos, antifúngicos o corticosteroides en los últimos tres meses, no haber usado antiséptico oral previo a la toma de muestra y la autorización y firma de la carta de consentimiento informado por parte de los padres o tutores del niño ${ }^{15}$.

\section{Obtención de muestras}

El estudio se diseñó con dos tomas de muestra, la primera, previa a la colocación de los aditamentos ortodónticos y otra, a los seis meses posteriores a su colocación. Las muestras se tomaron con un hisopo estéril, que se frotó rotatoriamente sobre la mucosa oral y el dorso de la lengua de cada uno de los pacientes, las que fueron sembradas en un tiempo no mayor de dos horas posterior a su toma en el Departamento de Microbiología Oral y Biología Molecular de la Facultad de Odontología de la Universidad Autónoma de Yucatán.

\section{Aislamiento e identificación de Candida}

Las muestras se cultivaron en Agar Dextrosa Sabouraud (ADS) (Difco $\left.{ }^{\circledR}\right)$ y presuntivamente identificadas en CHROMagar ${ }^{\circledR}$ Candida (BBL, Becton, Dickinson and Company) siguiendo las condiciones y criterios previamente descritos ${ }^{3,16,17}$.

Identificación molecular por reacción de polimerasa en cadena (RPC). Las colonias de las cepas presuntivas de Candida, fueron recuperadas en ADS (Difco $\left.{ }^{\circledR}\right)$ y resuspendidas en un vial conteniendo $500 \mu \mathrm{l}$ de agua destilada estéril, con la finalidad de extraer el ADN de las levaduras por el método de extracción rápida de ebullición-congelamiento, descrito previamente ${ }^{18}$. La identificación molecular de las cepas aisladas se llevó a cabo por el método de RPC múltiple, realizando dos paneles con tres especies cada uno, en el panel A ( $C$. tropicalis, $C$. glabrata y $C$. parapsilosis) y en el panel B (C. albicans, C. dubliniensis y C. krusei), las que fueron seleccionadas considerando el tamaño y migración de los fragmentos amplificados, y de acuerdo a los procedimientos previamente descritos ${ }^{3,19-21}$. Un par de oligonucleótidos fue utilizado por cada una de las seis especies estudiadas, así como uno específico para el género Candida (Tabla 1).

Tabla 1. Partidores utilizados para la identificación molecular de especies de Candida

\begin{tabular}{|c|c|c|c|c|c|}
\hline Especie & Nombre del partidor & Secuencia $\left(5^{\prime} \rightarrow 3^{\prime}\right)$ & Gen & $\mathrm{Pb}$ & Referencia \\
\hline \multirow[t]{2}{*}{ Género Candida } & RNAF & GCATATCAATAAGCGGAGGAAAAG & 25S ARNr & 610 & Hernández y cols, 2014³ \\
\hline & RNAR & GGTCCGTGTTTCAAGACG & & & \\
\hline \multirow[t]{2}{*}{ C. albicans } & CAL5 & TGTTGCTCTCTCGGGGGCGGCCG & $25 \mathrm{~S} A R N r$ & 175 & Hernández y cols, $2014^{3}$ \\
\hline & NL4CAL & AAGATCATTATGCCAACATCCTAGGTATAAA & & & \\
\hline \multirow[t]{2}{*}{ C. dubliniensis } & DUBF & GTATTTGTCGTTCCCCTTTC & ACT1 & 288 & Yang y cols, $2003^{19}$ \\
\hline & DUBR & GTGTTGTGTGCACTAACGTC & & & \\
\hline \multirow[t]{2}{*}{ C. krusei } & CKSF35 & GAGCCACGGTAAAGAATACACA & Topoisomerasa II & 227 & Estrada-Barraza y cols, $2011^{21}$ \\
\hline & CKSR57 & TTTAAAGTGACCCGGATACC & & & \\
\hline \multirow[t]{2}{*}{ C. glabrata } & CGL1 & TTATCACACGACTCGACACT & ITS1-ITS2 & 423 & Luo y Mitchell, $2002^{20}$ \\
\hline & CGL2 & CCCACATACTGATATGGCCTACAA & & & \\
\hline \multirow[t]{2}{*}{ C. tropicalis } & CTR1 & CAATCCTACCGCCAGAGGTTAT & ITS1-ITS2 & 357 & Luo y Mitchell, $2002^{20}$ \\
\hline & CTR2 & TGGCCACTAGCAAAATAAGCGT & & & \\
\hline \multirow[t]{2}{*}{ C. parapsilosis } & CPA3 & GCCAGAGATTAAACTCAACCAA & ITS1-ITS2 & 300 & Luo y Mitchell, $2002^{20}$ \\
\hline & CPA2 & CCTATCCATTAGTTTATACTCCGC & & & \\
\hline
\end{tabular}


Se utilizó una mezcla de reacción con un volumen final de $25 \mu$ conteniendo: 0,8 pmol de cada uno de los oligonucléotidos específicos, 2,5 $\mathrm{mM}$ de $\mathrm{MgCl}_{2}$, tampón RPC (10 mM de Tris- $\mathrm{HCl}, 10 \mathrm{mM}$ de $\mathrm{KCl}$ ), 0,2 $\mathrm{mM}$ de la mezcla de dNTPs, 2,5 U de Taq ADN polimerasa (Invitrogen) y $10 \mathrm{ng}$ del $\mathrm{ADN}$ en estudio. La reacción de amplificación se llevó a cabo con un ciclo inicial a $95^{\circ} \mathrm{C}$ durante seis min, seguido de 30 ciclos de 30 s a $94{ }^{\circ} \mathrm{C}$, 30 s a $58{ }^{\circ} \mathrm{C}$ y $30 \mathrm{~s}$ a $72{ }^{\circ} \mathrm{C}$, con una incubación final de diez min a $72{ }^{\circ} \mathrm{C}^{3,19,22}$.

Los fragmentos amplificados se visualizaron mediante la electroforesis en un gel de agarosa al 1,5\% en tampón TBE (Tris-Borato de sodio-EDTA) 1X, a un voltaje constante de corrida de $100 \mathrm{~V}$ durante $1 \mathrm{~h}$. El gel se tiñó con bromuro de etidio $(0,5 \mathrm{mg} / \mathrm{ml})$ durante $15 \mathrm{~min}$. Las bandas fueron visualizadas en un transiluminador de luz UV. El tamaño del fragmento del ADN amplificado fue determinado por comparación con un marcador de tamaño molecular de $100 \mathrm{pb}^{23}$. Se utilizaron las siguientes cepas de referencia: Candida albicans (ATCC 10231), Candida parapsilosis (ATCC 22019), Candida krusei (ATCC 14243), Candida glabrata (ATCC 90030), Candida tropicalis (ATCC 750) y Candida dubliniensis (ATCC MYA-646).

\section{Análisis estadístico}

La influencia del uso de la aparatología ortodóntica sobre la presencia de las especies de Candida en cavidad oral y tipo de aparatología usada, se realizó con la prueba estadística de $\chi^{2}$ con un nivel de confianza del $95 \%$.

\section{Resultados}

Se estudiaron 60 niños que iniciaron tratamiento con aparatología ortodóntica; 32 (53,3\%) fueron del género femenino y $28(46,7 \%)$ del masculino. El intervalo de edad fue de 4 a 10 años, siendo la edad promedio de 6,1 años. Asimismo, 28 (46,7\%) usaron aparatología fija y $32(53,3 \%)$ removible.

Del total de los pacientes estudiados, $9(15,0 \%)$ tuvieron cultivos positivos a Candida spp. en la mucosa bucal antes de iniciar el tratamiento y en el resto la pesquisa fue negativa. En la segunda muestra, a los seis meses de iniciado el tratamiento ortodóntico, se encontraron 28 $(46,7 \%)$ pacientes con cultivo positivo y $32(53,3 \%)$ con cultivos negativos a Candida spp. (Tabla 2).

Se encontró una influencia de la presencia de la aparatología ortodóntica sobre la ocurrencia de Candida spp. en la cavidad oral, con un valor estadísticamente significativo $(\mathrm{p}<0,001)$. Con la aparatología fija se incrementó de 3 a 18 cultivos positivos al paso del tiempo $(\mathrm{p}<0,05)$, a diferencia de la aparatología removible donde el incremento fue mínimo, de 6 a 10 cultivos positivos $(p>0,05)$.

\begin{tabular}{|c|c|c|c|c|}
\hline \multirow[t]{2}{*}{ Candida spp. } & \multicolumn{2}{|c|}{ Antes } & \multicolumn{2}{|c|}{ Después } \\
\hline & $\mathbf{n}$ & $\%$ & $\mathrm{n}$ & $\%$ \\
\hline Candida albicans & 5 & 8,3 & 11 & 18,4 \\
\hline Candida tropicalis & 2 & 3,3 & 12 & 20,0 \\
\hline Candida krusei & 1 & 1,7 & 3 & 5,0 \\
\hline Candida parapsilosis & 1 & 1,7 & 2 & 3,3 \\
\hline Total & 9 & 15,0 & 28 & 46,7 \\
\hline
\end{tabular}

Al analizar los resultados de los cultivos, antes y después del tratamiento ortodóntico, todos los pacientes con cultivo positivo al inicio del tratamiento, también lo fueron al final del mismo. De ellos, un paciente tuvo un cambio de especie de C. albicans por C. tropicalis. Considerando la aparición de una nueva especie como caso nuevo, se encontraron 20 niños que dieron cultivos positivos después del uso de aparatología ortodóntica. Se realizó la prueba estadística de $\chi^{2}$, encontrándose una diferencia estadísticamente significativa $(\mathrm{p}<0,0001)$.

Antes del tratamiento, $C$. albicans fue la especie prevalente $(8,3 \% ; 5 / 60)$, seguido de $C$. tropicalis $(3,3 \%$; $2 / 60), C$. krusei y $C$. parapsilosis $(1,7 \% ; 1 / 60)$.

A los seis meses de iniciado el tratamiento, C. tropicalis fue la especie más frecuente $(20,0 \% ; 12 / 60)$, luego C. albicans $(18,4 \% ; 11 / 60)$, C. krusei y C. parapsilosis (5,0 y 3,3\%, respectivamente).

En ninguno de los dos muestreos se encontraron cultivos positivos de C. dubliniensis o C. glabrata (Tabla 2).

\section{Discusión}

Se estudió la influencia del uso de la aparatología ortodóntica sobre la presencia de especies de Candida en la cavidad oral de pacientes odontológicos infantiles. Antes del inicio del tratamiento de ortodoncia, la prevalencia de portadores de Candida en niños fue de $15,0 \%$, resultado similar a lo reportado con anterioridad por otros autores ${ }^{10,24,25}$, pero muy inferior a lo reportado por Arzate y cols $(2004)^{26}$, quienes encontraron en una población sana pediátrica mexicana, una prevalencia de $43 \%$, y el hallazgo descrito por Hernández-Solís y cols (2008) en una comunidad rural del estado de Yucatán, México, de 58,2\% $\%^{27}$. Esta discrepancia pudiera deberse a que la población estudiada por nosotros fue previamente sometida a tratamiento odontológico, eliminando factores de riesgo o promotores de colonización por Candida spp., como lo son la placa bacteriana y caries. Por el contrario, concuerda con lo reportado por Gaitán-Cepeda y cols. 
(2012), en un grupo de 29 niños sanos de 4 a 13 años de edad incluidos en un programa de prevención de caries y salud bucal, quienes reportaron una frecuencia de Candida spp. de $10,3 \%{ }^{28}$.

Candida albicans fue la especie más frecuente entre los pacientes, previo al inicio de tratamiento, siendo 55,5\% del total de los cultivos positivos a Candida, seguida de C. tropicalis $(22,2 \%)$, similar a lo reportado en otros estudios $^{24,26,27,29}$.

Se observó un incremento significativo de 15,0 a 46,7\% de niños portadores de especies de Candida desde el inicio y a seis meses de iniciado el tratamiento ortodóntico, hallazgo similar a lo reportado por otros autores $9,15,30,31$.

El presente trabajo, presenta evidencias significativas que la instalación de aparatología ortodóntica puede predisponer a la colonización por especies de Candida, probablemente, debido a la interferencia que tienen en un adecuado cepillado y limpieza de la cavidad oral, como previamente se ha reportado ${ }^{15,32,33}$. La inadecuada limpieza bucal favorece la acumulación de placa dento-bacteriana debido a la presencia de microorganismos orales que influyen en cambios en la microbiota oral ${ }^{8,15,34}$. La teoría microecológica oral sostiene que existe un balance ecológico entre los microorganismos de la cavidad oral y el propio individuo, y éste se ve alterado cuando las condiciones del hospedero cambian. La colocación de aparatología ortodóntica no sólo influye en la acumulación de placa dento-bacteriana; también se ha asociado a incremento en la colonización microbiana, desmineralización del esmalte dental, cambios en la capacidad amortiguadora de la saliva e incluso inflamación en los tejidos periodontales ${ }^{8,9,15,30,35}$.

No se debe omitir, que los materiales como el acrílico y el metal utilizados en la aparatología ortodóntica presentan superficies rugosas y/o porosas que dan las condiciones adecuadas para la adherencia sobre la superficie de Candida, lo que, aunado a una higiene oral deficiente y otros factores de riesgo del hospedero, pueden facilitar un incremento en el número de microorganismos presentes en la cavidad oral $8,9,12,30$.

Cabe destacar que, en un paciente al que inicialmente se le encontró $C$. albicans, a los seis meses se aisló $C$. tropicalis, mismo fenómeno reportado por Khanpayeh $\mathrm{y} \operatorname{cols}^{8}$, lo que hace prestar mayor atención al riesgo de infecciones de diferentes especies asociadas al uso de aparatología ortodóntica, principalmente en niños inmunocomprometidos.

Se observó que la aparatología fija influyó en mayor presencia de Candida spp. en comparación con la removible, resultado similar a lo encontrado por Khanpayeh y cols., y otros autores ${ }^{8,33}$. Aunado a esto, Ronsani y cols., comprueban que los iones metálicos liberados de la degradación en boca de la aparatología ortodóntica pueden contribuir a la modulación de los factores de virulencia y aumento en la biomasa de Candida $^{36}$.

Por lo tanto, la aparatología ortodóntica y ortopédica, sea fija o removible, puede tener efectos perjudiciales sobre el control de la placa bacteriana, por lo que es de suma importancia que los pacientes estén informados de que esos aditamentos pueden servir como reservorio de diferentes microorganismos, haciendo hincapié en que se debe prestar especial atención a su higiene oral.

La colonización de Candida spp. en cavidad oral por uso de aparatología ortodóntica es capaz de causar condiciones inflamatorias sobre la mucosa que la soporta, y en estos casos es necesaria una desinfección eficaz de los mismos, para llevar a la mejoría del proceso inflamatorio. La desinfección eficaz por lo general puede reducir al mínimo la necesidad de terapia antifúngica, que a su vez minimiza el riesgo de la resistencia emergente a fármacos antifúngicos.

Los resultados del presente estudio, demostraron que el uso de aditamentos ortodónticos en la cavidad oral, influye en la ocurrencia de especies de Candida. Por lo que debe darse a los pacientes asesoramiento periódico e instrucciones sobre la higiene de la cavidad oral y de la aparatología, lo que resultará en un efecto preventivo sobre posibles colonizaciones de Candida spp. y probables procesos infecciosos, sobre todo, en pacientes que presenten condiciones apropiadas para su desarrollo.

\section{Resumen}

Introducción: El uso de aparatología ortodóntica crea condiciones favorables para el incremento de la placa bacteriana. Objetivo: Evaluar si el uso de aparatología ortodóntica influye en la presencia de especies de Candida en la cavidad oral, en un grupo de pacientes pediátricos. Material y Métodos: Se estudiaron 60 pacientes entre 4 y 10 años de edad, que requirieron tratamiento con aparatología ortodóntica. Se tomaron muestras de la cavidad oral al inicio del tratamiento ortodóntico y a los seis meses de uso. Las muestras fueron sembradas en Agar Dextrosa Sabouraud y CHROMagar ${ }^{\circledR}$ Candida. Las especies de Candida se confirmaron por RPC múltiple. Los resultados se analizaron con la prueba estadística de chi cuadrada. Resultados: La presencia de Candida fue mayor en las muestras tomadas a los seis meses de iniciado el tratamiento $(p<0,001)$. La especie más frecuente antes del tratamiento fue $C$. albicans $(8,3 \%)$ y después del tratamiento fue $C$. tropicalis $(20,0 \%)$. Conclusiones: El uso de aparatología ortodóntica influye en la presencia de especies de Candida, por lo que deben tomarse medidas preventivas para evitar su conversión en candidiasis oral, en especial, en pacientes inmunodeprimidos. 


\section{Referencias bibliográficas}

1.- Rueda-Gordillo F, Hernández-Solís S E. Prevalencia de Candida albicans aislada de la cavidad oral de pacientes con cáncer. Rev Odontol Latinoam 2008; 0: 38-41.

2.- Rueda-Gordillo F, Hernández-Solís S E, Ordoñez-Sánchez W, Villamil-Urzaiz J L, Godoy-Montañez C. Portadores de Candida oral en pacientes atendidos en una clínica dental de Tabasco, México. Rev Odontol Latinoam 2011; 3: 45-8.

3.- Hernández-Solís S E, Rueda-Gordillo F, Rojas-Herrera R A. Proteinase activity in Candida albicans strains isolated from the oral cavity of immunocompromised patients, with oral candidiasis and in healthy subjects. Rev Iberoam Micol 2014; 31: 137-40.

4.- Patel M, Shackleton J T, Coogan M M. Effect of antifungal treatment on the prevalence of yeasts in HIV-infected subjects. J Med Microbiol 2006; 55: 1279-84.

5.- Boriollo M F, Bassi R C, dos Santos Nascimento C M, Feliciano L M, Francisco S B, Barros L M, et al. Distribution and hydrolytic enzyme characteristics of Candida albicans strains isolated from diabetic patients and their non-diabetic consorts. Oral Microbiol Immunol 2009; 24: 437-50.

6.- Rammohan S N, Juvvadi S R, Gandikota C S, Challa P, Manne R, Mathur A. Adherence of Streptococcus mutans and Candida albicans to different bracket materials. J Pharm Bioallied Sci 2012; 4: S212-6.

7.- Goncalves e Silva C R, Oliveira L D, Leao M V, Jorge A O. Candida spp. adherence to oral epithelial cells and levels of IgA in children with orthodontic appliances. Braz Oral Res 2014; 28: 28-32.

8.- Khanpayeh E, Jafari A A, Tabatabaei Z. Comparison of salivary Candida profile in patients with fixed and removable orthodontic appliances therapy. Iran J Microbiol 2014; 6: 263-8.

9.- Hibino K, Wong R W, Hagg U, Samaranayake L P. The effects of orthodontic appliances on Candida in the human mouth. Int J Paediatr Dent 2009; 19: 301-8.

10.- Nevzatoglu S, Kücükkeles N, Kadir T. Frecuencia de la Candida albicans en niños que utilizan aparatos de ortodoncia removible. Rev Esp Ortod 2011; 41: 49-53.

11.- Mukumoto M, Ohshima T, Ozaki M, Konishi H, Maeda N, Nakamura Y. Effect of microbubbled water on the removal of a biofilm attached to orthodontic appliances-an in vitro study. Dent Mater J 2012; 31: 821-7.

12.- Saloom H F, Mohammed-Salih H S, Rasheed S F. The influence of different types of fixed orthodontic appliance on the growth and adherence of microorganisms (in vitro study). J Clin Exp Dent 2013; 5: e36-41.

13.- Arendorf T, Addy M. Candidal carriage and plaque distribution before, during and after removable orthodontic appliance therapy. J Clin Periodontol 1985; 12: 360-8.

14.- Brusca M I, Chara O, Sterin-Borda L, Rosa A C. Influence of different orthodontic brackets on adherence of microorganisms in vitro. Angle Orthod 2007; 77: 331-6.

15.- Arslan S G, Akpolat N, Kama J D, Özer T, Hamamci O. One-year follow-up of the effect of fixed orthodontic treatment on colonization by oral candida. J Oral Pathol Med 2008; 37: 26-9.

16.- Furlaneto-Maia L, Specian A F, Bizerra F C, de Oliveira M T, Furlaneto M C. In vitro evaluation of putative virulence attributes of oral isolates of Candida spp. obtained from elderly healthy individuals. Mycopathologia 2008; 166: 209-17.

17.- Rajkumari N, Mathur P, Xess I, Misra M C. Distribution of different yeasts isolates among trauma patients and comparison of accuracy in identification of yeasts by automated method versus conventional methods for better use in low resource countries. Indian J Med Microbiol 2014; 32: 391-7.

18.- De Baere T, Claeys G, Swinne D, Verschraegen $\mathrm{G}$, Muylaert A, Massonet C, et al. Identification of cultured isolates of clinically important yeast species using fluorescent fragment length analysis of the amplified internally transcribed rRNA spacer 2 region (ITS2). BMC Microbiol 2002; 2: 21.

19.- Yang C W, Barkham T M, Chan F Y, Wang Y. Prevalence of Candida species, including Candida dubliniensis, in Singapore. J Clin Microbiol 2003; 41: 472-4.

20.- Luo G, Mitchell T G. Rapid identification of pathogenic fungi directly from cultures by using multiplex PCR. J Clin Microbiol 2002; 40: 2860-5.

21.- Estrada-Barraza D, Dávalos Martínez A, FloresPadilla L, Mendoza-De Elías R, SánchezVargas L O. Comparison between conventional methods, Chrom Agar Candida (R) and PCR method for the identification of Candida species in clinical isolates. Rev Iberoam Micol 2011; 28: 36-42.

22.- Polacheck I, Strahilevitz J, Sullivan D, Donnelly S, Salkin I F, Coleman D C. Recovery of Candida dubliniensis from non-human immunodeficiency virus-infected patients in Israel. J Clin Microbiol 2000; 38: 170-4.

23.- Martins M, Henriques M, Ribeiro A P, Fernandes R, Goncalves V, Seabra A, et al. Oral Candida carriage of patients attending a dental clinic in Braga, Portugal. Rev Iberoam Micol 2010; 27: 119-24.

24.- Kaur A, Soodan P S, Soodan K S, Priyadarshni P. Evaluation of prevalence of Candida species in the root canals and oral cavity of children and adult patients. IOSR-JDMS. 2014; 13: 100-4.

25.- Sedgley C M, Samaranayake L P, Chan J C, Wei S H. A 4-year longitudinal study of the oral prevalence of enteric gram-negative rods and yeasts in Chinese children. Oral Microbiol Immunol 1997; 12: 183-8.

26.- Arzate M N, Sánchez V O, Calderón B L, Aquino G S, Gaitán C L. Prevalencia de portadores de especies de Candida en cavidad bucal en una población pediátrica. Rev Odont Mex 2004; 8: 107-11.

27.- Hernández-Solís S E, Rueda-Gordillo F, Pereira-Góngora J R, Villamil-Urzaiz J L. Frecuencia de portadores de C. albicans en un grupo de niños de una comunidad rural del estado de Yucatán. Rev Odontol Latinoam 2008; $0: 1-4$.

28.- Gaitán-Cepeda L A, Sánchez-Vargas L O, Pavia-Ruz N, Muñoz-Hernández R, VillegasHam J, Ceballos-Salobreña A. Candida bucal en niños mexicanos con VIH/SIDA, desnutrición o marginación social. Rev Panam Salud Pública 2012; 31: 48-53.

29.- Kadir T, Uygun B, Akyuz S. Prevalence of Candida species in Turkish children: relationship between dietary intake and carriage. Arch Oral Biol 2005; 50: 33-7.

30.- Zheng Y, Li Z, He X. Influence of fixed orthodontic appliances on the change in oral Candida strains among adolescents. J Dent Sci 2014; http://dx.doi.org/10.1016/j. jds.2014.02.001.

31.- Jabur S F. Influence of removable orthodontic appliance on oral microbiological status. J Fac Med Baghdad 2008; 50: 199-202.

32.- Kitada K, de Toledo A, Oho T. Increase in detectable opportunistic bacteria in the oral cavity of orthodontic patients. Int J Dent Hygiene 2009; 7: 121-5.

33.- Hagg U, Kaveewatcharanont P, Samaranayake Y H, Samaranayake L P. The effect of fixed orthodontic appliances on the oral carriage of Candida species and Enterobacteriaceae. Eur J Orthod 2004; 26: 623-9.

34.- Arikan V, Kizilci E, Ozalp N, Ozcelik B. Effects of fixed and removable space maintainers on plaque accumulation, periodontal health, candidal and Enterococcus faecalis carriage. Med Princ Pract 2015; 24: 311-7.

35.- Dar-Odeh N, Shehabi A, Al-Bitar Z, Al-Omari I, Badran S, Al-Omiri M, et al. Oral Candida colonization in patients with fixed orthodontic appliances: The importance of some nutritional and salivary factors. Afr J Microbiol Res 2011; 5: 2150-4.

36.- Ronsani M M, Mores Rymovicz A U, Meira T M, Trindade Grégio A M, Guariza Filho O, Tanaka O M, et al. Virulence modulation of Candida albicans biofilms by metal ions commonly released from orthodontic devices. Microb Pathog 2011; 51: 421-5. 